\title{
ON THE FEKETE-SZEGÖ PROBLEM FOR CLOSE-TO-CONVEX FUNCTIONS
}

\author{
WOLFRAM KOEPF
}

ABSTRACT. Let $S$ be the familiar class of normalized univalent functions in the unit disk. Fekete and Szegö proved the well-known result

$$
\max _{f \in S}\left|a_{3}-\lambda a_{2}^{2}\right|=1+2 e^{-2 \lambda /(1-\lambda)}
$$

for $\lambda \in[0,1]$. We consider the corresponding problem for the family $C$ of close-to-convex functions and get

$$
\max _{f \in C}\left|a_{3}-\lambda a_{2}^{2}\right|= \begin{cases}3-4 \lambda & \text { if } \lambda \in[0,1 / 3], \\ 1 / 3+4 /(9 \lambda) & \text { if } \lambda \in[1 / 3,2 / 3], \\ 1 & \text { if } \lambda \in[2 / 3,1] .\end{cases}
$$

As an application it is shown that ||$a_{3}|-| a_{2}|| \leq 1$ for close-to-convex functions, in contrast to the result in $S$

$$
\max _{f \in S}|| a_{3}|-| a_{2}||=1.029 \ldots
$$

1. Introduction. Let $S$ denote the family of univalent functions $f$ of the unit disk, normalized by

$$
f(z)=z+a_{2} z^{2}+a_{3} z^{3}+\cdots .
$$

Let $S t$ denote the subset of starlike functions, i.e. functions that have a starlike range with respect to the origin. Further let $C$ denote the family of close-to-convex functions, which have been introduced by Kaplan [4]. A function $f$, normalized by (1), is called close-to-convex if there exist a starlike function $g$ and a real number $\alpha$, such that

$$
\left.\operatorname{Re}\left(e^{i \alpha} z f^{\prime}(z) / g(z)\right)>0, \quad \alpha \in\right]-\pi / 2, \pi / 2[.
$$

It turns out that a function is close-to-convex if and only if it maps the unit disk univalently onto a domain whose complement is the union of half-lines, which are pairwise disjoint up to possibly equal tips (see $[\mathbf{6}-\mathbf{7}, \mathbf{1}]$ ).

A well-known function of this kind is the Koebe function $k$ with

$$
k(z)=\sum_{n=1}^{\infty} n z^{n}=\frac{z}{(1-z)^{2}}=\frac{1}{4}\left(\left(\frac{1+z}{1-z}\right)^{2}-1\right),
$$

which maps the unit disk onto the complement of the half-line ] $-\infty,-1 / 4]$, as the last representation shows.

Many extremal problems within the class $S$ are solved by the Koebe function. On the other hand, the Koebe function satisfies

$$
\left|a_{3}-\lambda a_{2}^{2}\right|=|3-4 \lambda|
$$

Received by the editors November 19, 1985 and, in revised form, May 30, 1986. 1980 Mathematics Subject Classification (1985 Revision). Primary 30C45, 30C50.

Key words and phrases. Close-to-convex functions, univalent functions. 
whereas Fekete and Szegö showed [3]

$$
\max _{f \in S}\left|a_{3}-\lambda a_{2}^{2}\right|=1+2 e^{-2 \lambda /(1-\lambda)}
$$

for $\lambda \in[0,1]$,

For $\lambda=0,1$ the Koebe function gives the maximum, but there is no $\left.\lambda_{0} \in\right] 0,1[$ such that the functional $\left|a_{3}-\lambda_{0} a_{2}^{2}\right|$ is maximized by $k$. We shall show that

$$
\max _{f \in C}\left|a_{3}-\lambda a_{2}^{2}\right|=3-4 \lambda
$$

for $\lambda \in[0,1 / 3]$, so that for close-to-convex functions the situation is quite different. This result implies furthermore that

$$
\max _{f \in C}|| a_{3}|-| a_{2}||=1
$$

in contrast to the known estimate in $S$,

$$
\max _{f \in S}|| a_{3}|-| a_{2}||=1.029 \ldots
$$

(see e.g. [2, Theorem 3.11]). Moreover we show that

$$
\max _{f \in C}\left|a_{3}-\lambda a_{2}^{2}\right|= \begin{cases}1 / 3+4 /(9 \lambda) & \text { if } \lambda \in[1 / 3,2 / 3], \\ 1 & \text { if } \lambda \in[2 / 3,1] .\end{cases}
$$

2. Preliminary results. Here we give some lemmas which will be used in the next section to solve the main problem.

Recall that a function $f$ is called close-to-convex of order $\beta$ if there exist a starlike function $g$ and a real number $\alpha$, such that

$$
\left|\arg \left(e^{i \alpha} z f^{\prime}(z) / g(z)\right)\right|<\beta \pi / 2 .
$$

LEMMA 1 (see [5, Lemma 1]). Let $f \in C$. Then the function $h$, defined by

$$
h^{\prime}(z)=\left(f^{\prime}\left(z^{2}\right)\right)^{1 / 2}, \quad h(0)=0
$$

is an odd close-to-convex function of order $1 / 2$.

LEMMA 2 (see [8, p. 166, formula (10)]). Let $p(z)=1+p_{1} z+p_{2} z^{2}+\cdots$ and $\operatorname{Re} p>0$. Then

$$
\left|p_{2}-p_{1}^{2} / 2\right| \leq 2-\left|p_{1}\right|^{2} / 2 \text {. }
$$

LEMMA 3. Let $g(z)=z+b_{2} z^{2}+b_{3} z^{3}+\cdots \in S t$. Then

$$
\left|b_{3}-\lambda b_{2}^{2}\right| \leq \max \{1,|3-4 \lambda|\}
$$

which is sharp for the Koebe function $k$ if $|\lambda-3 / 4| \geq 1 / 4$ and for $\left(k\left(z^{2}\right)\right)^{1 / 2}=$ $z /\left(1-z^{2}\right)$ if $|\lambda-3 / 4| \leq 1 / 4$.

ProOF. Because $g \in S t$, the function

$$
z g^{\prime}(z) / g(z)=1+b_{2} z+\left(2 b_{3}-b_{2}^{2}\right) z^{2}+\cdots=1+\dot{p}_{1} z(3)+p_{2} z^{2}+\cdots
$$

has positive real part, so that $\left|p_{2}-\frac{1}{2} p_{1}^{2}\right| \leq 2-\left|p_{1}\right|^{2} / 2$ by Lemma 2 . Let now $\lambda \in \mathbf{C}$. Then by (3) we have

$$
\begin{aligned}
\left|b_{3}-\lambda b_{2}^{2}\right| & =\frac{1}{2}\left|p_{2}+(1-2 \lambda) p_{1}^{2}\right|=\frac{1}{2}\left|p_{2}-\frac{1}{2} p_{1}^{2}+\left(\frac{3}{2}-2 \lambda\right) p_{1}^{2}\right| \\
& \leq \frac{1}{2}\left(2-\frac{1}{2}\left|p_{1}\right|^{2}+\left|\frac{3}{2}-2 \lambda\right|\left|p_{1}\right|^{2}\right)^{2} .
\end{aligned}
$$


If now $|\lambda-3 / 4| \leq \frac{1}{4}$, then

$$
\left|b_{3}-\lambda b_{2}^{2}\right| \leq \frac{1}{2}\left(2-\frac{1}{2}\left|p_{1}\right|^{2}+\frac{1}{2}\left|p_{1}\right|^{2}\right)=1 .
$$

On the other hand, if $|\lambda-3 / 4| \geq \frac{1}{4}$, then we use $\left|p_{1}\right| \leq 2$ (see e.g. [8, Corollary 2.3]), and get

$$
\begin{aligned}
\left|b_{3}-\lambda b_{2}^{2}\right| & \leq 1+\frac{1}{2}\left(\left|\frac{3}{2}-2 \lambda\right|-\frac{1}{2}\right)\left|p_{1}\right|^{2} \\
& \leq 1+|3-4 \lambda|-1=|3-4 \lambda| .
\end{aligned}
$$

3. Main results. The first step of the solution of the Fekete-Szegö problem for close-to-convex functions is the special case $\lambda=1 / 3$.

THEOREM 1. Let $f(z)=z+a_{2} z^{2}+a_{3} z^{3}+\cdots \in C$. Then $\left|a_{3}-\frac{1}{3} a_{2}^{2}\right| \leq \frac{5}{3}$.

ProOF. Let $f \in C$. Then by Lemma 1 the function $h$, defined by (2), is an odd close-to-convex function of order $1 / 2$.

For such functions, the author gave sharp bounds on the coefficients (see [5, Theorem 1]), in particular, the fifth coefficient of $h$ is bounded in modulus by $1 / 2$. On the other hand the fifth coefficient of $h$ is given by $\frac{3}{10}\left(a_{3}-\frac{1}{3} a_{2}^{2}\right)$, which implies the result.

The next corollary follows easily from the theorem using $\left|a_{2}\right| \leq 2$ (see e.g. [2, Theorem 2.2]).

COROllary 1. Let $\lambda \in[0,1 / 3]$. Then

$$
\max _{f \in C}\left|a_{3}-\lambda a_{2}^{2}\right|=3-4 \lambda .
$$

The maximum is attained by the Koebe function.

Another consequence of the theorem is the following result about successive coefficients of close-to-convex functions.

COROLlary 2. Let $f \in C$. Then ||$a_{3}|-| a_{2}|| \leq 1$.

PROOF. It is well known that $\left|a_{2}\right|-\left|a_{3}\right| \leq 1$ for all $f \in S$ (see e.g. [2, Theorem 3.11]). Moreover, if $\left|a_{2}\right| \leq 1$, then also $\left|a_{3}\right|-\left|a_{2}\right| \leq 1$ (see e.g. [2, proof of Theorem 3.11]). Now let $f \in C$ and $\left|a_{2}\right| \in[1,2]$. Then Theorem 1 implies that

$$
\begin{aligned}
\left|a_{3}\right|-\left|a_{2}\right| & \leq\left|a_{3}-\frac{1}{3} a_{2}^{2}\right|+\frac{1}{3}\left|a_{2}\right|^{2}-\left|a_{2}\right| \\
& \leq \frac{5}{3}+\frac{1}{3}\left|a_{2}\right|^{2}-\left|a_{2}\right| \leq 1,
\end{aligned}
$$

as $\left|a_{2}\right|$ is in the above range.

The following notation will be used throughout the paper. For $f(z)=z+a_{2} z^{2}+$ $a_{3} z^{3}+\cdots \in C$ there is a representation of the form

$$
f^{\prime}(z)=\frac{g(z)}{z} \cdot \tilde{p}(z)
$$

with some function $g(z)=z+b_{2} z^{2}+b_{3} z^{3}+\cdots \in S t$ and some function $\tilde{p}(z)=$ $1+\tilde{p}_{1} z+\tilde{p}_{2} z^{2}+\cdots$ such that $\left.\operatorname{Re}\left(e^{i \alpha} \tilde{p}(z)\right)>0, \alpha \in\right]-\pi / 2, \pi / 2[$. Then the function $p(z)=1+p_{1} z+p_{2} z^{2}+\cdots$, defined by

$$
\tilde{p}_{n}=\cos \alpha \cdot e^{-i \alpha} \cdot p_{n}, \quad n \in \mathbf{N},
$$

has positive real part. Comparing coeffficients in (4) we get

$$
3 a_{3}=b_{3}+\tilde{p}_{1} b_{2}+\tilde{p}_{2}, \quad 2 a_{2}=b_{2}+\tilde{p}_{1},
$$


so that

$$
a_{3}-\lambda a_{2}^{2}=\frac{1}{3}\left(b_{3}-\frac{3}{4} \lambda b_{2}^{2}\right)+\frac{1}{3}\left(\tilde{p}_{2}-\frac{3}{4} \lambda \tilde{p}_{1}^{2}\right)+\tilde{p}_{1} b_{2}\left(\frac{1}{3}-\lambda / 2\right) .
$$

Now we consider the case $\lambda=2 / 3$.

THEOREM 2. Let $f(z)=z=a_{2} z^{2}+a_{3} z^{3}+\cdots \in C$. Then $\left|a_{3}-\frac{2}{3} a_{2}^{2}\right| \leq 1$.

ProOF. From (6) it follows that

$$
\left|a_{3}-\frac{2}{3} a_{2}^{2}\right| \leq \frac{1}{3}\left|b_{3}-\frac{1}{2} b_{2}^{2}\right|+\frac{1}{3}\left|\tilde{p}_{2}-\frac{1}{2} \tilde{p}_{1}^{2}\right| .
$$

From (5) we get

$$
\begin{aligned}
\tilde{p}_{2}-\frac{1}{2} \tilde{p}_{1}^{2} & =\cos \alpha \cdot e^{-i \alpha}\left(p_{2}-\frac{1}{2} \cos \alpha \cdot e^{-i \alpha} p_{1}^{2}\right) \\
& =\cos \alpha \cdot e^{-i \alpha}\left(p_{2}-\frac{1}{2} p_{1}^{2}+\mu p_{1}^{2}\right),
\end{aligned}
$$

where $|2 \mu|^{2}=\left|1-\cos \alpha \cdot e^{-i \alpha}\right|^{2}=\sin ^{2} \alpha$. Now we get with the aid of Lemmas 2 and 3 that

$$
\begin{aligned}
\left|a_{3}-\frac{2}{3} a_{2}^{2}\right| & \leq \frac{1}{3}+\frac{1}{3} \cos \alpha\left(2-\frac{\left|p_{1}\right|^{2}}{2}\right)+\frac{1}{3} \cos \alpha|\sin \alpha| \frac{\left|p_{1}\right|^{2}}{2} \\
& \leq 1-\cos \alpha \frac{\left|p_{1}\right|^{2}}{6}(1-|\sin \alpha|) \leq 1 .
\end{aligned}
$$

An easy consequence using $\left|a_{3}-a_{2}^{2}\right| \leq 1$ is

COROLlaRY 3. Let $\lambda \in[2 / 3,1]$. Then

$$
\max _{f \in C}\left|a_{3}-\lambda a_{2}^{2}\right|=1 \text {. }
$$

The maximum is attained by the function $\left(k\left(z^{2}\right)\right)^{1 / 2}$.

We remark that Theorem 2 provides a direct proof of $\left|a_{3}\right|-\left|a_{2}\right| \leq 1$ for $\left|a_{2}\right| \leq 3 / 2$ (compare with the proof of Corollary 2), namely

$$
\begin{aligned}
\left|a_{3}\right|-\left|a_{2}\right| & \leq\left|a_{3}-\frac{2}{3} a_{2}^{2}\right|+\frac{2}{3}\left|a_{2}\right|^{2}-\left|a_{2}\right| \\
& \leq 1+\frac{2}{3}\left|a_{2}\right|^{2}-\left|a_{2}\right| \leq 1
\end{aligned}
$$

if $\left|a_{2}\right| \in[0,3 / 2]$.

It remains to consider the case $\lambda \in] 1 / 3,2 / 3[$.

THEOREM 3. Let $\lambda \in] 1 / 3,2 / 3[$. Then

$$
\max _{f \in C}\left|a_{3}-\lambda a_{2}^{2}\right|=\frac{1}{3}+\frac{4}{9 \lambda} \text {. }
$$

The maximum is attained by the function $f$, which is defined by

$$
f^{\prime}(z)=\frac{1}{(1-z)^{2}} \cdot\left(t \frac{1+z}{1-z}+(1-t) \frac{1+z^{2}}{1-z^{2}}\right), \quad f(0)=0,
$$

where $t=2 /(3 \lambda)-1$.

PROOF. Consider equation (6). We use the estimate $\left|b_{3}-\frac{3}{4} \lambda b_{2}^{2}\right| \leq 3(1-\lambda)$, which comes from Lemma 3 , further equations (5) and $\left|b_{2}\right| \leq 2$, getting

$$
\left|a_{3}-\lambda a_{2}^{2}\right| \leq 1-\lambda+\frac{\cos \alpha}{3}\left|p_{2}-\frac{3}{4} \lambda \cos \alpha \cdot e^{-i \alpha} p_{1}^{2}\right|+\cos \alpha\left(\frac{2}{3}-\lambda\right)\left|p_{1}\right| .
$$


Writing $\frac{3}{4} \lambda \cos \alpha \cdot e^{-i \alpha}=\frac{1}{2}-\mu$, we have

$$
|2 \mu|^{2}=\left|1-\frac{3}{2} \lambda \cos \alpha \cdot e^{-i \alpha}\right|^{2}=1-\left(3 \lambda-\frac{9}{4} \lambda^{2}\right) \cos ^{2} \alpha,
$$

which implies with the aid of Lemma 2 that

$$
\left|p_{2}-\frac{3}{4} \lambda \cos \alpha \cdot e^{-i \alpha} p_{1}^{2}\right| \leq 2+\frac{\left|p_{1}\right|^{2}}{2}\left(\sqrt{1-\left(3 \lambda-\frac{9}{4} \lambda^{2}\right) \cos ^{2} \alpha-1}\right)
$$

so that-using the notations $y:=\cos \alpha$ and $p:=\left|p_{1}\right|$-it follows that

$$
\begin{aligned}
\left|a_{3}-\lambda a_{2}^{2}\right| & \leq 1-\lambda+y\left(\frac{2}{3}+\frac{p^{2}}{6}\left(\sqrt{1-\left(3 \lambda-\frac{9}{4} \lambda^{2}\right) y^{2}}-1\right)+p\left(\frac{2}{3}-\lambda\right)\right) \\
& =: F_{\lambda}(p, y) .
\end{aligned}
$$

For further simplification we shall use the notation $\gamma:=2-3 \lambda$.

Now we shall show that $F_{\lambda}$ attains its maximum value for $(p, y) \in[0,2] \times[0,1]$ at the point $(4 /(3 \lambda)-2,1)$. Observe that

$$
F_{\lambda}\left(\frac{4}{3 \lambda}-2,1\right)=\frac{1}{3}+\frac{4}{9 \lambda}
$$

Suppose now that $F_{\lambda}$ attains its maximum value at an interior point $\left(p_{0}, y_{0}\right) \in$ ] $0,2[\times] 0,1\left[\right.$. Then the partial derivates $\partial F_{\lambda} / \partial p$ and $\partial F_{\lambda} / \partial y$ must vanish at $\left(p_{0}, y_{0}\right)$. The equality $\left(\partial F_{\lambda} / \partial p\right)\left(p_{0}, y_{0}\right)=0$ gives the relation

$$
\sqrt{1-\left(3 \lambda-\frac{9}{4} \lambda^{2}\right) y_{0}^{2}}-1=-\frac{\gamma}{p_{0}}
$$

so that

$$
\left(3 \lambda-\frac{9}{4} \lambda^{2}\right) y_{0}^{2}=\frac{2 \gamma}{p_{0}}-\frac{\gamma^{2}}{p_{0}^{2}} .
$$

Now, $\left(\partial F_{\lambda} / \partial y\right)\left(p_{0}, y_{0}\right)=0$ implies

$$
\frac{2}{3}+\frac{\gamma p_{0}}{6}=\frac{p_{0}^{2}\left(2 \gamma / p_{0}-\gamma^{2} / p_{0}^{2}\right)}{6\left(1-\gamma / p_{0}\right)}
$$

so that, by solving the quadratic equation for $p_{0}$, we get

$$
\gamma p_{0}=2\left(1-\sqrt{1-\gamma^{2}}\right)
$$

Therefore, at $\left(p_{0}, y_{0}\right)$ the value of $F_{\lambda}$ becomes, using (8) and (9),

$$
\begin{aligned}
F_{\lambda}\left(p_{0}, y_{0}\right) & =1-\lambda+y\left(\frac{2}{3}+\frac{1}{3}\left(1-\sqrt{1-\gamma^{2}}\right)\right) \\
& \leq \frac{4+\gamma-\sqrt{1-\gamma^{2}}}{3}
\end{aligned}
$$

because $y \leq 1$.

Since $\lambda \in] 1 / 3,2 / 3$ [, the number $\gamma$ lies between 0 and 1 so that there is some $\delta \in$ ]0, $\pi / 2\left[\right.$ with $\gamma=\cos \delta$ and $\sqrt{1-\gamma^{2}}=\sin \delta$. The evident inequality $1<\cos \delta+\sin \delta$ 
implies

$$
\begin{aligned}
2-\cos \delta<1+\sin \delta \\
\quad \Rightarrow(2-\cos \delta)(1-\sin \delta)<1-\sin ^{2} \delta=\cos ^{2} \delta \\
\Rightarrow(2-\gamma)\left(1-\sqrt{1-\gamma^{2}}\right)<\gamma^{2} \\
\Rightarrow(2-\gamma)\left(4+\gamma-\sqrt{1-\gamma^{2}}\right)<6-\gamma \\
\Rightarrow \frac{4+\gamma-\sqrt{1-\gamma^{2}}}{3}<\frac{1}{3}+\frac{4}{3(2-\gamma)}=\frac{1}{3}+\frac{4}{9 \lambda} .
\end{aligned}
$$

Thus, using (7) and (10), we get a contradiction to our assumption that $F_{\lambda}$ attains its maximum value at $\left(p_{0}, y_{0}\right)$, so that the maximum must be attained at a boundary point.

In both cases $y=0$ and $p=0$ an easy computation shows that the maximal value (7) is not attained. If $y=1$ we have

$$
F_{\lambda}(p, 1)=: G_{\lambda}(p)=\frac{5}{3}-\lambda+\left(\frac{2}{3}-\lambda\right) p-\frac{\lambda}{4} p^{2} .
$$

Because $G_{\lambda}(2)=3-4 \lambda$ is not maximal, the local maximum at $p=4 /(3 \lambda)-2$ - given by $d G_{\lambda}(p) / d p=0$-is global. This leads to the maximal value (7).

Now it remains to prove that

$$
F_{\lambda}(p, y) \leq \frac{1}{3}+\frac{4}{9 \lambda}
$$

for $p=2, y \in] 0,1[$. This statement is equivalent to

$$
H_{\gamma}(y):=2 y\left(\sqrt{1-\left(1-\frac{\gamma^{2}}{4}\right) y^{2}}+\gamma\right) \leq \frac{4}{2-\gamma}-\gamma
$$

for $\gamma=2-3 \lambda \in] 0,1\left[\right.$. Because we already know that $H_{\gamma}(y) \leq 4 /(2-\gamma)-\gamma$ when $y \in\{0,1\}$, it suffices to show (11) for points with $d H_{\gamma}(y) / d y=0$. This leads to

$$
\left(1-\frac{\gamma^{2}}{4}\right) y^{2}=\frac{4-\gamma^{2}+\gamma \sqrt{8+\gamma^{2}}}{8}
$$

Observe that $0 \leq y \leq 1$ when (12) is satisfied. Squaring inequality (11) and substituting (12) gives the following inequality:

$$
\begin{gathered}
4\left(\frac{4-\gamma^{2}+\gamma \sqrt{8+\gamma^{2}}}{8}\right)\left(\frac{\sqrt{8+\gamma^{2}}-\gamma}{4}+\gamma\right)^{2} \\
\leq\left(1-\frac{\gamma^{2}}{4}\right)\left(\frac{4}{2-\gamma}-\gamma\right)^{2} .
\end{gathered}
$$

It remains to prove (13). A lengthy calculation gives-after multiplying with the number $(2-\gamma)$, which is positive-the equivalent version

$$
\begin{aligned}
\gamma(2-\gamma)\left(8+\gamma^{2}\right)^{3 / 2} & \leq(4+2 \gamma)\left(4-2 \gamma+\gamma^{2}\right)^{2}-(2-\gamma)\left(8+20 \gamma^{2}-\gamma^{4}\right) \\
& =48-24 \gamma-24 \gamma^{2}+28 \gamma^{3}-2 \gamma^{4}+\gamma^{5}
\end{aligned}
$$


The right-hand side turns out to be positive:

$$
\begin{aligned}
& 48-24 \gamma-24 \gamma^{2}+28 \gamma^{3}-2 \gamma^{4}+\gamma^{5} \\
& >28 \gamma^{3}-2 \gamma^{4}+\gamma^{5}=\gamma^{3}\left(28-2 \gamma+\gamma^{2}\right)>\gamma^{3}\left(26+\gamma^{2}\right) \geq 0,
\end{aligned}
$$

so that equivalently, squaring again

$$
\gamma^{2}(2-\gamma)^{2}\left(8+\gamma^{2}\right)^{3} \leq\left(48-24 \gamma-24 \gamma^{2}+28 \gamma^{3}-2 \gamma^{4}+\gamma^{5}\right)^{2}
$$

A further lengthy computation gives the equivalent reformulation

$$
\begin{aligned}
\gamma^{8}- & 2 \gamma^{7}+17 \gamma^{6}-12 \gamma^{5}-70 \gamma^{4}+184 \gamma^{3}-118 \gamma^{2}-72 \gamma+72 \\
& =(1-\gamma)^{2}\left(\gamma^{6}+16 \gamma^{4}+20 \gamma^{3}-46 \gamma^{2}+72 \gamma+72\right) \\
& =(1-\gamma)^{2}\left(\gamma^{6}+16 \gamma^{4}+20 \gamma^{3}+26 \gamma^{2}+72 \gamma(1-\gamma)+72\right) \geq 0
\end{aligned}
$$

which is trivially true. This finishes the proof of the inequality

$$
\left|a_{3}-\lambda a_{2}^{2}\right| \leq \frac{1}{3}+\frac{4}{9 \lambda} \text {. }
$$

From our considerations it follows that equality holds if $b_{2}=2$ and $b_{3}=3$ (so that $g$ is a rotation of $k$ ), and if $\alpha=0, p_{2}=2$, and $p_{1}=4 /(3 \lambda)-2$; the function

$$
\tilde{p}(z)=t\left(\frac{1+z}{1-z}\right)+(1-t)\left(\frac{1+z^{2}}{1-z^{2}}\right), \quad t=\frac{2}{3 \lambda}-1,
$$

satisfies these conditions, which makes the result sharp.

\section{REFERENCES}

1. A. Bielecki and Z. Lewandowski, Sur un théorème concernant les fonctions univalentes linéairement accessibles de M. Biernacki, Ann. Polon. Math. 12 (1962), 61-63.

2. P. L. Duren, Univalent functions, Springer-Verlag, New York-Berlin-Heidelberg-Tokyo, 1983.

3. M. Fekete and G. Szegö, Eine Bemerkung über ungerade schlichte Functionen, J. London Math. Soc. 8 (1933), 85-89.

4. W. Kaplan, Close-to-convex schlicht functions, Michigan Math. J. 1 (1952), 169-185.

5. W. Koepf, Coefficients of symmetric functions of bounded boundary rotation (to appear).

6. Z. Lewandowski, Sur l'identité de certaines classes de fonctions univalentes. I, Ann. Univ. Mariae Curie-Skłodowska 12 (1958), 131-146.

7. __ Sur l'identité de certaines classes de fonctions univalentes. II, Ann. Univ. Mariae CurieSkłodowska 14 (1960), 19-46.

8. Ch. Pommerenke, Univalent functions, Vandenhoeck \& Ruprecht, Göttingen, 1975.

Freie Universität Berlin, Fachbereich Mathematik, Arnimallee 3, 1000 BerLiN 33, Federal REPUBLIC OF GERMANY 